Palacios-Martínez, G. E., \& Núñez-Zamora, I. (2021) Posibilidades de promoción social para los pescadores costeros de Playas del Coco, Guanacaste, Costa Rica, utilizando el Turismo Marino Sostenible. Revista de Biología Tropical, 69(Suppl. 2), S29-S35. https://doi. org/10.15517/rbt.v69iS2.48740

Biologín Tropical

https://doi.org/10.15517/rbt.v69iS2.48740

\title{
Posibilidades de promoción social para los pescadores costeros de Playas del Coco, Guanacaste, Costa Rica, utilizando el Turismo Marino Sostenible
}

\author{
Gerardo Emigdio Palacios-Martínez1; (D) https://orcid.org/0000-0003-1132-0870 \\ Ileana Núñez-Zamora ${ }^{2}$; (D) https://orcid.org/0000-0003-4080-1024 \\ 1. Fundación Promar, OCEANews, CCT, San Pedro, San José, Costa Rica; gerardopalacios2009@gmail.com \\ 2. Escuela de Trabajo Social, Universidad Libre de Costa Rica, San José, Costa Rica; nu-ezileana@hotmail.com
}

Recibido 30-I-2021. Corregido 13-III-2021. Aceptado 06-V-2021.

\author{
ABSTRACT \\ Possibilities of social promotion for the coastal inhabitants of Playas del Coco, \\ Guanacaste, Costa Rica, using Sustainable Marine Tourism
}

\begin{abstract}
Introduction: Fishermen in Playas del Coco, Guanacaste, Costa Rica, are vulnerable due to the mismanagement of fishing resources and illegal fishing; excluding them from benefits granted by the State. This means that they do not have many options that allow them to grow both economically and socially.

Objective: Determine a social promotion strategy for people in social exclusion in Playas del Coco.

Methods: From February to September 2018, participatory action and critical research were applied, through semi-structured and open interview, and participant observation to members of the Development Association, community leaders and fishermen in the area, ranging in age between 17 and 40 years old.

Results: Theoretical and practical bases were established for an interdisciplinary work between social work, tourism and marine biology through the intervention strategy for fishermen of Playas del Coco based on the Socio-educational Promotional Model and the current situation of the inhabitants was documented.

Conclusions: The incursion of social science in environmental issues is viable, by social promotion of the inhabitants of Playas del Coco developing sustainable marine tourism through a socio-educational model.
\end{abstract}

Key words: Social theories; human rights; entrepreneurship; conservation; marine resources.

Parte de la problemática de algunos pescadores no agremiados en Playas del Coco Guanacaste, Costa Rica, se debe a la desorganización laboral, un mínimo apoyo institucional, redes de apoyo casi nulas y a la ausencia de programas especializados para un desarrollo integral que les de acceso a oportunidades de promoción social y actividades económicas rentables. Los pescadores artesanales en Playas del Coco son vulnerables debido al mal manejo y gestión de los recursos pesqueros y la pesca ilegal; excluyéndoles de los beneficios otorgados por el Estado. Esto incide en que no cuenten con opciones que les permita crecer tanto a nivel económico como social (Solorzano, Solís, \& Ayales, 2016).

Dicha problemática es tratada en varias investigaciones, pero no involucran al turismo ni a la biología marina en un trabajo interdisciplinario como lo sugiere el presente trabajo. Se 
ha establecido la pertinencia del trabajo social en el desarrollo comunitario participativo en zonas rurales y el uso responsable de los recursos naturales a través de estrategias socioeducativas que brinden sostenibilidad ambiental, social y económica (Chávez, Gálvez, \& Guidos, 2014). Por otro lado, existe escasa información sobre las medidas gubernamentales con relación a las zonas costeras de Costa Rica, los procesos de conservación ambiental y desarrollo sostenible. Se establece que los gobiernos locales no tienen las competencias o recursos para dar las respuestas necesarias en materia social y ambiental (Aguilar \& Arroyo, 2014; Larios 2014; Kung, 2015).

Algunos estudios identifican las necesidades de las comunidades con relación a la protección del ambiente, el desarrollo comunal, la economía de las poblaciones costeras con exclusión social y su relación con el trabajo social (Bonilla et al., 2015; Duque, 2014; Santos, 2015). Hay autores que relacionan las alteraciones ambientales con el tipo de producción y su efecto sobre la salud pública y las soluciones respectivas (Bonilla et al., 2015; Larios, 2014). Desde la intervención de trabajo social, en esta problemática es posible promover soluciones a través de sus teorías y modelos, permitiendo una acción más participativa del sujeto de investigación.

Teorías relacionadas: La Teoría Crítica aplicada por medio del trabajo social motiva al sujeto intervenido a ser más crítico de su propia realidad, empoderarse para que se organice y participe en las decisiones de carácter público (Larios, 2014), así como para que considere formas sociales democráticas para modificar sus realidades. Complementando esta teoría, están los Derechos Humanos, que elevan la calidad de vida de los ciudadanos incluyendo agrupaciones excluidas socialmente (Rodríguez, 2011). Dentro de las alternativas de promoción social se incluye el Turismo Marino Sostenible (TMS): prácticas turísticas marinas de calidad, solidarias y responsables, con encadenamientos productivos con otros sectores, creando oportunidades laborales dignas aprovechando los recursos marinos, sin comprometer el recurso para las generaciones futuras. En Playas del Coco, el TMS incluye: buceo, pesca deportiva, observación de fauna y flora marina, entre otros (Rodríguez-Fonseca, CuberoPardo, Palacios-Martínez, Ramírez-Hernández, \& Hernández-Blanco, 2010). Esta situación facilita los emprendimientos productivos de este tipo de turismo (Núñez, 2018).

La Teoría de las Necesidades Humanas promueve que la comunidad asuma una participación activa por medio del TMS para obtener una promoción social que solvente su problemática, posibilitando la acción de los actores en espacios de incidencia en el desarrollo sostenible y mejoramiento de calidad de vida (Núñez, 2018). El trabajo social interviene a los seres humanos cuyas circunstancias dificultan su desarrollo digno y autónomo comparado con otros seres humanos en el ámbito social (Bermejo, 2000).

\section{Modelo Socioeducativo Promocional:} Desde el Modelo Socioeducativo Promocional, se plantea una posibilidad de ejecución que interviene y promueve la transformación de realidades de las comunidades estudiadas (Jiménez, 2000). Este modelo promueve que la comunidad mejore su calidad de vida, por medio del análisis de sus contextos, la capacitación, la concienciación, la movilización de recursos personales y grupales, que permita tomar decisiones en pro del mejoramiento de su calidad de vida (Molina \& Romero, 2004).

Este trabajo pretende diseñar una estrategia de promoción social para los pescadores en exclusión social de Playas del Coco, Guanacaste, con el fin de definir un protocolo de intervención que les permita implementar el Turismo Marino Sostenible, incidiendo en su desarrollo con igualdad de oportunidades, equidad de género, tolerancia ante la diversidad y la reciprocidad en el trato; estableciendo una interacción proactiva con las instituciones comunales y del Estado. 


\section{MATERIALES Y MÉTODOS}

Se realizaron entrevistas semi estructuradas y abiertas para recolectar información de fuente primaria y analizar las posibilidades de promoción social que tienen las personas de Playas del Coco, Guanacaste, utilizando el TMS. También se utilizó la observación participante, la cual documenta la cotidianeidad de los entrevistados, obteniendo información sobre sus comportamientos y de los acontecimientos, tal y como se producen (Kawulich, 2005).

Doce entrevistados aceptaron un Consentimiento Informado, por lo que se les invitó a participar en la investigación para confirmar sus aportes en este proceso. La entrevista analizada se encuentra en los anexos de Núñez (2018). La selección de la población se dio mediante el abordaje a la Asociación de Desarrollo local, los líderes comunales y habitantes de la zona con edades comprendidas entre los 17 y 40 años que se dedican a la pesca artesanal, sin distinción de género. Las entrevistas se realizaron de febrero a septiembre del 2018, en la zona costera de Playas del Coco, Distrito de Sardinal, Cantón de Carrillo, Provincia de Guanacaste, Costa Rica.

Se aplicó la investigación Acción Participativa como metodología de empoderamiento y transformación de la realidad social, además, para promover procesos participativos, donde las personas pueden influir en su propio cambio. Se desarrolló un análisis a través de la Teoría Crítica, por medio de procesos de autorreflexión, con el fin de motivar la transformación de su realidad, generando una estrategia de intervención para el mejoramiento de la calidad de vida y alcanzar un desarrollo sostenible.

\section{RESULTADOS}

Este artículo analiza doce encuestados por Núñez (2018) que se caracterizan como la población pesquera de Playas del Coco, en exclusión social, con bajos recursos económicos. De los encuestados, $67 \%$ respondió no tener ningún conocimiento con relación a los Derechos Humanos y 33\% dijo tener derecho al trabajo, a la libre navegación, igualdad de oportunidades y derecho a la inclusión.

Con respecto a las necesidades prioritarias, el $100 \%$ comentó la necesidad de obtener suministros para poder pescar y aunque todos consideran necesaria la educación la dejaron de lado por la necesidad de cooperar con los ingresos del hogar. En cuanto a la participación ciudadana, $83 \%$ de los entrevistados respondieron que no participan en procesos comunales, ya que no poseen los recursos para tener sus obligaciones al día.

De igual forma, las personas externaron sus apreciaciones con respecto al potencial del ambiente marino local y su conservación a través de las entrevistas realizadas. $100 \%$ de los participantes tenían conocimientos empíricos sobre las especies marinas locales, 58\% tenían experiencia sobre la pesca deportiva y $83 \%$ conocía las especies marinas locales con potencial de observación turística, como la observación de delfines y ballenas y el "snorkeling". Con relación al emprendimiento, $100 \%$ de los participantes expresaron el de deseo de iniciar un negocio propio.

Sobre exclusión e inclusión social, 100\% no manejó el concepto, pero todos, una vez explicado, se sintieron excluidos de todo tipo de beneficio, principalmente por no poder pagar las cuotas y estar al día en las obligaciones legales de su actividad. Muchas de las ayudas, según expresaron, el Gobierno las centraliza en el Golfo de Nicoya y los excluye a ellos. Todos los entrevistados respondieron que no se les valora como pescadores artesanales, ni aprecian su trabajo como una herencia cultural.

A partir de la discusión generada a través de la aplicación de las entrevistas y la observación participante, se realizó un análisis y se diseñó la siguiente estrategia de intervención para aplicar a pescadores artesanales de Playas del Coco, basada en el Modelo Socioeducativo Promocional:

1. Concienciación sobre sus posibilidades de promoción social para un desarrollo sostenible desde sus percepciones, en los espacios de capacitación de la Asociación 
de Desarrollo, la Municipalidad, el Cantón o La Cámara de Pescadores y Armadores de Guanacaste.

2. Realización de talleres socioeducativos promocionales para promover la conformación de grupos sociales capacitados que les permita autogestionar su desarrollo, implementando alianzas con la Asociación de Desarrollo de la comunidad, las universidades, el INA, profesionales independientes y ONG's presentes en la zona.

3. Desarrollo de charlas educativas, donde visualicen sus derechos desde las políticas de promoción y protección social que ofrece el Estado, por medio de la Municipalidad del Cantón, de INCOPESCA y de profesionales independientes de trabajo social.

4. Capacitación a la comunidad por parte de las entidades financieras u organismos competentes que ofrecen posibilidades económicas para alcanzar un desarrollo sostenible. Involucrando instituciones como IMAS y el INA.

5. Incursión en turismo marino sostenible para desarrollar emprendimientos, con la colaboración del INA, Municipalidad y profesionales en biología marina tomando en cuenta sus posibilidades y recursos.

\section{DISCUSIÓN}

Derechos Humanos: $67 \%$ de los entrevistados no tienen una visión clara de cuáles son sus derechos y en qué consisten, posiblemente debido a que, en la mayoría de los casos, sus niveles educativos no trascienden de la primaria (Morux \& Murillo, 2013). De igual forma el $67 \%$ de ellos, confunden los derechos con beneficios que desearían que se les otorgaran, esto debido a la falta de presencia de instituciones que les informen al respecto, así como la falta de interés de parte de los individuos por informarse (MINAE, 2017).

Necesidades humanas: Se comparó esta base teórica con los entrevistados en Playas del Coco, los cuales manifestaron necesidades individuales y sociales. El 100\% de entrevistados comentó la necesidad de obtener suministros para poder pescar, todos priorizaron sus necesidades según las carencias en sus familias. Para algunos de estos hogares, la educación está en segundo plano, debido a la necesidad prioritaria de laborar para colaborar con la economía familiar (Heller, 1986; Larios, 2014). Esto muestra la importancia de promover mayores opciones de empleo, tomando en cuenta que la preparación técnica o académica es fundamental para el logro de oportunidades laborales de calidad, aunque se advirtió que para esta comunidad, no ha sido una de sus prioridades; se ubica a estas familias en una posición de desventaja para el acceso a fuentes de empleo mejor remuneradas. Se considera necesario promover en esta comunidad procesos socioeducativos que les permita priorizar adecuadamente sus necesidades, cambiar sus realidades y ofrecer a sus familias un desarrollo integral (Roig, 2009; Rodríguez, 2011). También se contempla la posibilidad que la Municipalidad gestione convenios o redes interinstitucionales para ofrecer capacitaciones técnicas que promuevan mejores condiciones de vida con relación a las necesidades evidenciadas en la comunidad (Larios, 2014; Kung, 2015). Se observaron otras necesidades, como el acceso al seguro social y la promoción social de sus familias (Heller, 1986; Larios, 2014).

Participación ciudadana: $83 \%$ de esta población no ejerce la participación ciudadana y se aísla por no poseer los recursos económicos necesarios para formar parte de las organizaciones de pescadores artesanales, ya que por su vulnerabilidad dejan de pagar sus permisos o sus licencias. Esto les sitúa en la informalidad laboral para satisfacer sus necesidades inmediatas, dejando de aportar al desarrollo integral de la comunidad (Rodríguez, 2011). Los entrevistados identificaron la deficiente distribución de recursos llevada a cabo por las organizaciones de pesca artesanal y del Estado, como el principal obstáculo que limita su participación. Se sugiere mejorar la calidad de vida de los pescadores mediante procesos de 
participación concertada con el Gobierno local, las instituciones públicas y privadas, así como con las organizaciones de la sociedad civil para el logro de un desarrollo sostenible (Bonilla et al., 2015; Granados, 2009).

Turismo Marino Sostenible, potencial y conservación: Todos los encuestados opinaron que este turismo tiene el potencial de contribuir con el desarrollo sostenible en Playas del Coco, debido a que todos en un $100 \%$ dijeron conocer empíricamente las especies marinas locales. Si se usa este potencial turístico de manera adecuada, se logrará una mejor conservación de sus recursos naturales, ya que no serán sacrificados sino preservados en beneficio de todos (Ramírez, 2015). Es viable desarrollar este tipo de turismo debido a las potencialidades que ya posee la población dedicada a la pesca artesanal, la cual tiende a comprender la importancia de proteger el medio ambiente. Esta comunidad sabe que a esta zona llegan turistas dispuestos a pagar por servicios de guiado marino, por lo que se recomienda valorar las oportunidades laborales que ofrece esa alternativa (Rodríguez-Fonseca et al., 2010).

Emprendimiento: Para implementar este tipo de emprendimiento los habitantes costeros poseen las habilidades para llevar turistas a visitar los diferentes ambientes marinos de sus alrededores, $100 \%$ dijeron querer iniciar algún tipo de emprendimiento, esto les ofrecería oportunidades de superación, debido a la variedad de posibilidades existentes (RodríguezFonseca et al., 2010).

Promoción social: Se considera que la promoción social es necesaria para que los pescadores de Playas del Coco mejoren su economía, beneficiando a toda la comunidad, aprovechando los recursos marinos, haciendo un uso responsable de estos recursos para generar fuentes de empleo sostenibles, capacitándose y conociendo sus derechos, a partir de proyectos que el Gobierno ofrece para convertirse en promotores de cambio de su propia realidad (Jiménez, 2000; Núñez, 2018; Sobrado, 1997).
Exclusión e inclusión social: Los pescadores en Playas del Coco sufren de exclusión social, ya que algunos operan la pesca ilegalmente. Esto genera un círculo vicioso, ya que las ayudas de las instituciones y organismos como la Cámara de Pescadores y Armadores de Guanacaste exigen que cuenten con sus permisos al día. Esta situación deja desprotegida a una parte de esta población que no cuentan con las mismas condiciones, promoviendo que la desigualdad aumente. Según algunos de los entrevistados hay corrupción dentro de las organizaciones, lo cual genera desconfianza para formar parte de las mismas, las ayudas no se reparten de forma justa, provocando que un sector de la población quede por fuera de los beneficios que pudieran recibir. Para alcanzar la inclusión social esta población debe empoderarse, organizarse y formar parte de procesos locales de desarrollo, para que aporten y ejecuten soluciones a sus necesidades (Núñez, 2018).

Acciones institucionales para la promoción social, económica y cultural: Los pescadores artesanales de Playas del Coco deben tener un acceso igualitario a políticas de protección y promoción que el Estado costarricense ofrece a personas en vulnerabilidad. Algunos de estos habitantes costeros están excluidos, perdiendo los beneficios que el Estado imparte para darles la oportunidad de surgir (ONU, 1987). En el tema económico, estos pescadores se encuentran desprotegidos, ya que el subsidio por el tiempo de veda de pesca aplica solo para pescadores del Golfo de Nicoya y los deja por fuera ya que ellos pertenecen al Golfo de Papagayo, y, aunque tienen limitaciones, no califican para las ayudas que proporciona el IMAS o estas son insuficientes (Núñez, 2018).

Conclusiones: La incursión del trabajo social en temas ambientales en coordinación con el turismo y la biología marina es viable por medio de la promoción social, la organización comunal, el trabajo cooperativo y el apoyo institucional. Los pescadores de Playas del Coco están anuentes a aplicar una estrategia 
de promoción social, utilizando el turismo marino como oportunidad laboral para mejorar su calidad de vida, por medio de un modelo socioeducativo promocional para un desarrollo sostenible. Finalmente, el acceso a programas de capacitación y promoción social es limitado, ya que no existe la presencia efectiva de instituciones estatales que generen estos espacios.

Declaración de ética: los autores declaran que todos están de acuerdo con esta publicación y que han hecho aportes que justifican su autoría; que no hay conflicto de interés de ningún tipo; y que han cumplido con todos los requisitos y procedimientos éticos y legales pertinentes. Todas las fuentes de financiamiento se detallan plena y claramente en la sección de agradecimientos. El respectivo documento legal firmado se encuentra en los archivos de la revista.

\section{AGRADECIMIENTOS}

A las pescadoras y a los pescadores artesanales de Playas del Coco, que con su amabilidad y gentileza abrieron un espacio en sus jornadas laborales y sus hogares para que este proyecto fuese posible.

\section{RESUMEN}

Introducción: Los pescadores en Playas del Coco, Guanacaste, Costa Rica, son vulnerables por el mal manejo y gestión de los recursos pesqueros y la pesca ilegal, excluyéndose de los beneficios otorgados por el Estado. Esto incide en que no cuenten con opciones que les permita crecer tanto a nivel económico como social.

Objetivo: Determinar una estrategia de promoción social para las personas en exclusión social de Playas del Coco.

Métodos: De febrero a septiembre 2018, se aplicó la Acción Participativa y la investigación Crítica, por medio de la entrevista semi estructurada y abierta, y la observación participante a miembros de la Asociación de Desarrollo, líderes comunales y pescadores de la zona, con edades entre los 17 y 40 años.

Resultados: Se establecieron las bases teórico prácticas para un trabajo interdisciplinario entre trabajo social, turismo y biología marina por medio de la estrategia de intervención para los pescadores artesanales de Playas del Coco basada en el Modelo Socioeducativo Promocional y se documentó la situación actual de los habitantes de esta región.

Conclusiones: La incursión del trabajo social en temas ambientales es viable, promocionando socialmente a los habitantes de Playas del Coco usando el turismo marino sostenible a través del modelo socioeducativo promocional.

Palabras clave: teorías sociales; derechos humanos; emprendimiento; conservación; recursos marinos.

\section{REFERENCIAS}

Aguilar, D., \& Arroyo, R. (2014). El amargo sabor de la piña: Movimiento socioambiental en el Caribe costarricense (Tesis para optar por el grado de Licenciatura en Trabajo Social). Universidad de Costa Rica, San José, Costa Rica.

Bermejo, F. (2000). Ética de las profesiones: Trabajo Social. Bilbao: Editorial Desclee.

Bonilla, E., Camacho, M., Granados, M., Montoya, J., Rodríguez, J., \& Vargas, N. (2015). Reconstrucción de la política ambiental en Costa Rica en materia de agua, cobertura forestal y energía en el periodo de 1994- 2010: Un análisis critico desde el Trabajo Social (Seminario para optar por el grado de Licenciatura en Trabajo Social). Universidad de Costa Rica, San José, Costa Rica.

Chávez, D., Gálvez, A., \& Guidos, D. (2014). Consultoría sobre elementos necesarios para la implementación de los proyectos de la Asociación de Desarrolladores y Promotores Turísticos Costero Marino (PROMAR) en los municipios de San Luis Talpa, Jiquilisco, Jucuarán, Intipucá y Conchagua bajo condiciones viables para su sostenibilidad (Tesis Maestro en Consultoría Empresarial). Universidad de El Salvador, San Salvador, El Salvador.

Duque, G. (2014). Trabajo social y ambiente: La importancia de incluir el tema ambiental en la formación de Trabajadores/as Sociales (Tesis para obtener el título de Maestría en Estudios Socioambientales). Facultad Latinoamericana de Ciencias Sociales, Sede Ecuador, Quito, Ecuador.

Granados, L. (2009). Participación social en el desarrollo local, dentro de la reforma estatal costarricense: la experiencia del Cantón de Oreamuno (Licenciatura en Trabajo Social). Universidad de Costa Rica, San José, Costa Rica.

Heller, A. (1986). Teoría de las necesidades en Marx. Barcelona, España: Ediciones Península.

Jiménez, A. (2000) La promoción social y su significado académico. Revista ABRA de la Universidad Nacional de Costa Rica, 21(30), 101-108.

Kawulich, B. B. (2005). La observación participante como método de recolección de datos. Fórum Qualitative 
Social Research, 6(2), Artículo 43. http://diverrisa. es/uploads/documentos/LA-OBSERVACION-PARTICIPANTE.pdf

Kung, J. (2015). La política social en el escenario de los municipios: perspectiva actual y nuevos desafios en torno a las manifestaciones de pobreza urbana y desigualdad en el cantón central de San José (Tesis para optar por la Licenciatura en Trabajo Social). Universidad de Costa Rica, San José, Costa Rica.

Larios, I. (2014). Trabajo social y saneamiento ambiental en la aldea El Terrero, Municipio de La Gomera en el Departamento de Escuintla (Tesis para optar por la Licenciatura en Trabajo Social). Universidad de San Carlos de Guatemala, Ciudad de Guatemala, Guatemala.

MINAE. (2017). Informe de estado del ambiente 2017. San José, Costa Rica: Ministerio de Ambiente y Energía.

Molina, M, \& Romero, M. (2004). Modelos de Intervención Asistencial, Socioeducativo y Terapéutico en Trabajo Social. San José, Costa Rica: Editorial de la Universidad de Costa Rica.

Morux, L., \& Murillo, J. (2013). El trabajo en el mar: situación del trabajador pesquero en Costa Rica (Tesis de Licenciatura). Universidad de Costa Rica, San José, Costa Rica.

Núñez, I. (2018). Posibilidades de promoción social para las personas de la zona costera de Playas del Coco, Guanacaste, utilizando el Turismo Marino como herramienta para un desarrollo sostenible, durante el periodo de febrero a setiembre 2018 (Tesis de Licenciatura). Universidad Libre de Costa Rica, Santa Cruz, Costa Rica.
ONU. (1987) Informe de la Comisión Mundial sobre el Medio Ambiente y el Desarrollo. Nota del Secretario General. https://undocs.org/es/A/42/427

Ramírez, G. (2015). La gestión del turismo sostenible: El caso español (Trabajo Fin de Grado en Turismo). Universidad de Cádiz, Cádiz, España.

Rodríguez-Fonseca, J., Cubero-Pardo, P., Palacios-Martínez, G., Ramírez-Hernández, P., Hernández-Blanco, M. (2010). Memoria del plan piloto para un modelo de turismo marino sostenible (TMS) en Costa Rica. San José, Costa Rica: Fundación Promar, TNC, HSI.

Rodríguez, V. (2011). Manual autoformativo sobre acceso a la justicia y derechos económicos, sociales y culturales. San José, Costa Rica: Instituto Interamericano de Derechos Humanos.

Roig, L. (2009). La gestión de problemas ambientales y el Trabajo Social Comunitario a nivel local. Su incidencia en la capacitación de actores locales en la comunidad "La Cabaña" del municipio Pinar del Río (Tesis para optar al título de Master en Desarrollo Social). Universidad de Pinar del Río, Pinar del Río, Cuba.

Santos, E. (2015). Trabajo social en la conservación del medio ambiente como área de acción profesional a nivel institucional (Tesis para optar por la Licenciatura en Trabajo Social). Universidad de San Carlos de Guatemala, Ciudad de Guatemala, Guatemala.

Sobrado, M. (1997) Promoción social. Periódico La Nación. https://www.nacion.com/opinion/promocion-social/ S6NYCAQM35HXPN73UA3QYUBWWY/story/

Solórzano, E., Solís, V., \& Ayales, I. (2016). Empleo rural decente en el sector de pesca artesanal y de pesca semiindustrial en Costa Rica: Caso de estudio. Roma, Italia: FAO. 